\title{
LETTER \\ Enhanced Derivation of Model Parameters for Cross-Component Linear Model (CCLM) in VVC
}

\author{
Yong-Uk YOON ${ }^{\dagger}$, Do-Hyeon PARK ${ }^{\dagger}$, Nonmembers, and Jae-Gon $\mathrm{KIM}^{\dagger \mathrm{a})}$, Member
}

\begin{abstract}
SUMMARY Cross-component linear model (CCLM) has been recently adopted as a chroma intra-prediction tool in Versatile Video Coding (VVC), which is being developed as a new video coding standard. CCLM predicts chroma components from luma components through a linear model based on assumption of linear correlation between both components. A linear model is derived from the reconstructed neighboring luma and chroma samples of the current coding block by linear regression. A simplified linear modeling method recently adopted in the test model of VVC (VTM) 3.0 significantly reduces computational complexity of deriving model parameters with considerable coding loss. This letter proposes a method of linear modeling to compensate the coding loss of the simplified linear model. In the proposed method, the model parameters which are quite roughly derived in the existing simplified linear model are refined more accurately using individual method to derive each parameter. Experimental results show that, compared to VTM 3.0, the proposed method gives $0.08 \%, 0.52 \%$ and $0.55 \%$ Bjotegaard-Delta (BD)-rate savings, for $\mathrm{Y}, \mathrm{Cb}$ and $\mathrm{Cr}$ components, respectively, in the All-Intra (AI) configuration with negligible computational complexity increase.

key words: JVET, VVC, chroma intra prediction, CCLM
\end{abstract}

\section{Introduction}

The Joint Video Experts Team (JVET) has been working on the development of next generation of video coding standard called Versatile Video Coding (VVC) [1]. In the current test model of VVC, VTM 3.0 [2], the chroma components of the coding block are predicted by various predefined modes including Cross-Component Linear Model (CCLM). Crosscomponent prediction techniques have been studied for a long time, and CCLM had been considered in the development of High Efficiency Video Coding (HEVC) and its performance had been verified [3]-[6].

CCLM basically assumes that luma and chroma components in the coding unit are linearly correlated. In other words, if the current chroma coding block is coded by CCLM mode, a linear model is derived from the neighboring luma and chroma samples by a linear regression. Then the chroma coding block is predicted by the reconstructed luma block with the derived linear model. Although CCLM was not adopted in the HEVC [7], CCLM came back in VTM 2.0 due to the significant improvement on chroma coding efficiency. However, the CCLM adopted in VTM 2.0 [8] had a large computational complexity to derive a linear model by linear regression, then the greatly simplified

\footnotetext{
Manuscript received March 5, 2019.

Manuscript revised August 13, 2019.

Manuscript publicized October 30, 2019.

${ }^{\dagger}$ The authors are with Korea Aerospace University, Deogyanggu, Goyang-si, Gyeonggi-do, 10540 Korea.

a)E-mail: jgkim@kau.ac.kr

DOI: 10.1587/transinf.2019EDL8045
}

CCLM has been adopted in VTM 3.0 [9].

\section{Cross-Component Linear Model (CCLM)}

CCLM adopted in VTM 2.0[8] consists of Linear Model (LM) mode and Multi-Directional LM (MDLM) mode. LM mode derives a linear model using available neighboring samples, regardless of prediction mode of luma, whereas MDLM mode derives a linear model using the left or above reference samples depending on prediction mode of luma. If CCLM is used, the current chroma block is predicted from the reconstructed luma block as:

$$
\operatorname{Pred}_{C}(i, j)=\alpha \times \operatorname{Rec}_{L}^{\prime}(i, j)+\beta,
$$

where $\operatorname{Pred}_{C}$ and $\operatorname{Rec}_{L}^{\prime}$ represent the predicted chroma samples and the reconstructed down-sampled luma samples in the current coding block, respectively; $(i, j)$ is the coordinate of a sample in the coding block. The linear parameters $\alpha$ and $\beta$ are derived by minimizing mean squared error (MSE) between neighboring reconstructed down-sampled luma samples and chroma samples as follows:

$$
\begin{aligned}
\alpha & =\frac{N \cdot \sum\left(\operatorname{Rec}_{C} \cdot \operatorname{Rec}_{L}^{\prime}\right)-\sum \operatorname{Rec}_{L}^{\prime} \cdot \sum \operatorname{Rec}_{C}}{N \cdot \sum\left(\operatorname{Rec}_{L}^{\prime} \cdot \operatorname{Rec}_{L}^{\prime}\right)-\sum \operatorname{Rec}_{L}^{\prime} \cdot \sum \operatorname{Rec}_{L}^{\prime}} \\
\beta & =\frac{\sum \operatorname{Rec}_{C}-\alpha \cdot \sum \operatorname{Rec}_{L}^{\prime}}{N},
\end{aligned}
$$

where $\operatorname{Rec}_{C}$ and $\operatorname{Rec}_{L}^{\prime}$ are the reconstructed chroma samples and down-sampled luma samples around the current coding block, respectively. $N$ is the total number of neighboring reference samples. Figure 1 shows the locations of the left and above reference samples involved in the calculation, marked as grey circles.

In VTM 3.0, to reduce computational complexity of
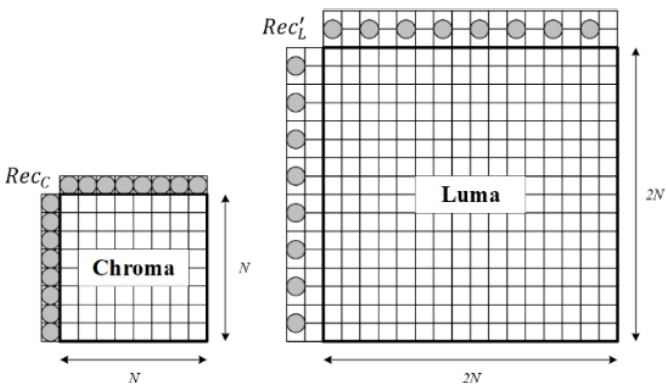

Fig. 1 Locations of the samples used to derive $\alpha$ and $\beta$ 
calculating linear regression, $\alpha$ and $\beta$ are derived in a simplified way. In other words, $\alpha$ and $\beta$ are derived from a straight line connecting the minimum and maximum values of the luma samples used for the derivation as shown in Fig. 2, where the $x$-axis and the $y$-axis represents the value of the luma and chroma samples, respectively. The points $A$ and $B$ correspond to the minimum and maximum value of the luma samples. In this way, $\alpha$ and $\beta$ are calculated as follows:

$$
\begin{aligned}
& \alpha=\frac{y_{B}-y_{A}}{x_{B}-x_{A}} \\
& \beta=y_{A}-\alpha \cdot x_{A}
\end{aligned}
$$

The simplified CCLM significantly reduces computational complexity of linear parameters derivation but has drawbacks in the representation of the overall distribution of neighboring samples which result in considerable coding loss. Because the parameters are derived just with the minimum and maximum values of the luma samples only, regardless of the distribution of the neighboring samples. Examples shown in Fig. 3 would be typical cases of bad fitting. When the reference samples are divided into two groups with less correlation (e.g., the left samples and above samples as shown in Fig. 3 (a)), some samples are not well fitted into a single linear model. In addition, the offset $\beta$ derived as (5) cannot refine the model to fit the distribution of samples. As shown in Fig. $3(\mathrm{~b})$, when the point $A\left(x_{A}, y_{A}\right)$ is placed at a position outside of the overall distribution, the derived linear model is deviated from the given distribution. In this letter, we propose a linear modeling to address such drawbacks in modelling of the existing method.

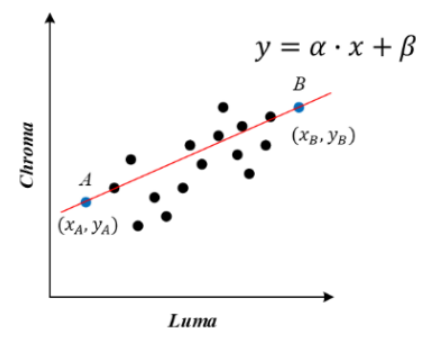

Fig. 2 The straight line connecting minimum and maximum luma values

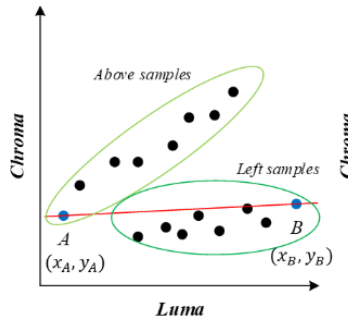

(a)

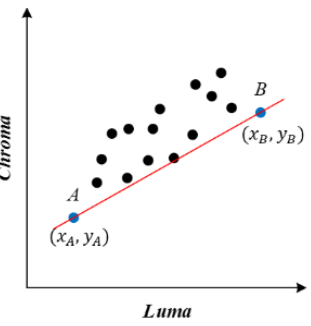

(b)
Fig. 3 Bad-fitting cases of the simplified linear parameter derivation. (a) An example of inappropriate linear model, (b) an example of linear model deviated from distribution

\section{Proposed Enhanced Derivation of Linear Model}

The proposed derivation of linear model is defined for each parameter of $\alpha$ and $\beta$. In order to obtain $\alpha$, not only the minimum and maximum values of the luma samples but also those of the chroma samples are considered. As shown in Fig. 4, all reference samples exist within the rectangle enclosed by the maximum and minimum values of luma and chroma, and $\alpha$ is defined as the slope of the diagonal of the rectangle as:

$$
\alpha= \begin{cases}\frac{\max \text { Chroma }-\min \text { Chroma }}{\max \text { Luma-min Luma }}, & \text { if }(4) \geq 0 \\ \frac{\min \text { Chroma }-\max \text { Chroma }}{\max \text { Luma }-\min \text { Luma }}, & \text { otherwise }\end{cases}
$$

Figure 4 shows an example of derived $\alpha$. The proposed method of obtaining $\alpha$ is applied when both the left and above reference samples are available. When either of the left and above reference samples is not available, $\alpha$ is derived by (4).

Then, the offset $\beta$ is determined by the average of the reference samples as:

$$
\beta=\frac{\sum \operatorname{Rec}_{C}}{N}-\alpha \cdot \frac{\sum \operatorname{Rec}_{L}^{\prime}}{N}
$$

The proposed method of deriving $\beta$ is applied regardless of whether the reference samples are available on both the left and above or not. Figure 5 shows an example of the proposed linear model derivation with the obtained $\alpha$ and $\beta$.

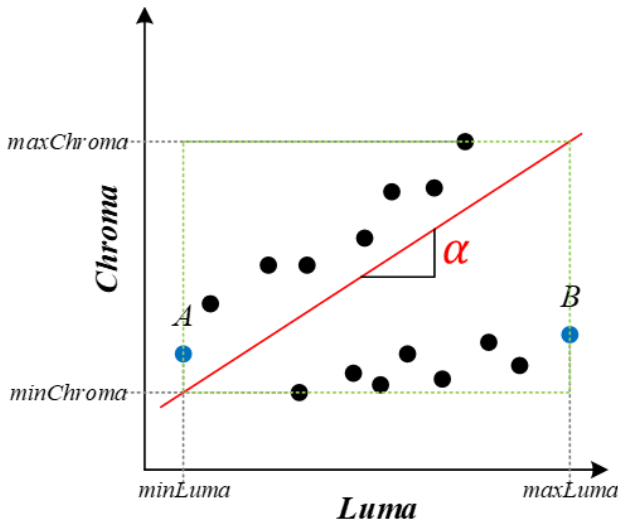

Fig. 4 An example of the obtained $\alpha$

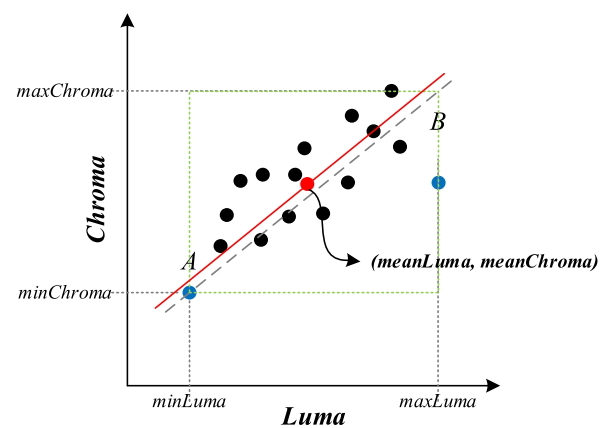

Fig.5 An example of the proposed linear model derivation 
The grey dotted line is a linear model having the derived slope of $\alpha$ without offset $\beta$, and the straight line marked in red is finally derived linear model refined with offset $\beta$.

The proposed method for computing the luma and chroma averages given by (7) adds negligible computational complexity of $2 \mathrm{~N}$ additions and two divisions, which can be implemented by shift operations, compared with the existing simplified method.

\section{Experimental Results}

The proposed method is implemented on top of VTM 3.0 and evaluated in the All-Intra (AI) encoding configuration according to the JVET common test conditions [10], [11]. Table 1 shows the performance results of the proposed method over VTM 3.0, which give $0.08 \%, 0.52 \%$ and $0.55 \%$ Bjotegaard-Delta (BD)-rate savings on average for $\mathrm{Y}, \mathrm{Cb}$ and $\mathrm{Cr}$ components, respectively, in the AI mode.

Table 2 shows the relationship between the complexity and BD-rate performance compared with the existing methods (CCLM of VTM 2.0 and VTM 3.0). VTM 3.0 reduced the computation quantity by $9 \mathrm{~N}+4$ compared to VTM 2.0 , and showed $0.11 \%, 0.48 \%$, and $-0.02 \%$ performance change for $\mathrm{Y}, \mathrm{Cb}$, and $\mathrm{Cr}$, respectively. On the other hand, the proposed method reduces the computation quantity by $7 \mathrm{~N}+2$ and shows the performance change of $0.03 \%$, $-0.04 \%$, and $-0.59 \%$ for $\mathrm{Y}, \mathrm{Cb}$, and $\mathrm{Cr}$, respectively. Therefore, the proposed method recovers the coding loss in the VTM 3.0 [9] and shows the gain especially in Cr component by adding some calculations. The comparisons show that the proposed method can achieve a better trade-off between coding efficiency and complexity.

Table 1 Performance of the proposed method over VTM 3.0

\begin{tabular}{|c|c|c|c|}
\hline \multirow{2}{*}{ Class } & \multicolumn{3}{|c|}{ Over VTM 3.0 in AI } \\
\cline { 2 - 4 } & $\mathrm{Y}$ & $\mathrm{U}$ & $\mathrm{V}$ \\
\hline \hline Class A1 & $-0.19 \%$ & $-0.33 \%$ & $-0.41 \%$ \\
\hline Class A2 & $-0.07 \%$ & $-0.30 \%$ & $-0.28 \%$ \\
\hline Class B & $-0.04 \%$ & $-0.74 \%$ & $-0.83 \%$ \\
\hline Class C & $-0.07 \%$ & $-0.72 \%$ & $-0.85 \%$ \\
\hline Class E & $-0.02 \%$ & $-0.29 \%$ & $-0.10 \%$ \\
\hline \hline Overall & $-0.08 \%$ & $-0.52 \%$ & $-0.55 \%$ \\
\hline
\end{tabular}

Table 2 Complexity and performance comparison

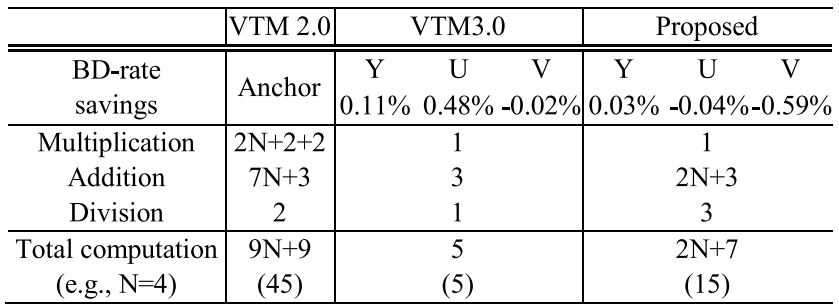

\section{Conclusions}

This letter proposed a method of linear modeling for CCLM to improve the existing simplified linear model which is recently adopted in VTM 3.0. In the proposed method, individual method is devised to derive each model parameters, $\alpha$ and $\beta$, which results in a more accurate model by refining the existing method. Experimental results demonstrated that the proposed method gives $0.08 \%, 0.52 \%$ and $0.55 \%$ BD-rate savings in average over VTM 3.0, for $\mathrm{Y}, \mathrm{Cb}$ and $\mathrm{Cr}$ components, respectively, in the AI mode with negligible complexity increase. Since CCLM is a promising coding tool for chroma components with efficient prediction, it is necessary to devise a method of model parameters derivation with lightweight computation while keeping the coding performance.

\section{Acknowledgments}

This work was partly supported by Institute for Information \& Communications Technology Promotion (IITP) grant funded by the Korea government (MSIT) (no. 20170-00486).

\section{References}

[1] B. Bross, J. Chen, and S. Liu, "Versatile video coding (draft 3)," 12th JVET Meeting, Doc. JVET-L1001, Oct. 2018.

[2] J. Chen, Y. Ye, and S. Kim, "Algorithm description for versatile video coding and test model 3 (VTM 3)," 12th JVET Meeting, Doc. JVET-L1002, Oct. 2018.

[3] S.H. Lee and N.I. Cho, "Intra prediction method based on the linear relationship between the channels for YUV 4:2:0 intra coding," Proc. IEEE Int. Conf. Image Process. (ICIP), pp.1037-1040, Nov. 2009.

[4] J. Kim, S.-W. Park, and B.-M. Jeon, "Intra chroma prediction using inter channel correlation," 2nd JCTVC Meeting, Doc. JCTVC-B021, July 2010.

[5] J. Chen et al., "Chroma intra prediction by scaled luma samples using integer operations," 3rd JCTVC Meeting, Doc. JCTVC-C206, Oct. 2010.

[6] C. Gisquet and E. Francois, "Model correction for cross-channel chroma prediction," Proc. Data Compression Conf. (DCC), pp.23-32, March 2013.

[7] G.J. Sullivan, J.-R. Ohm, W.-J. Han, and T. Wiegand, "Overview of the High Efficiency Video Coding (HEVC) Strandard," IEEE Trans. Circuits Syst. Video Technol., vol.22, no.12, pp.1649-1668, Dec. 2012.

[8] J. Chen, Y. Ye, and S. Kim, "Algorithm description for versatile video coding and test model 2 (VTM 2)," 11th JVET Meeting, Doc. JVET-K1002, July 2018.

[9] G. Laroche, J. Taquet, C. Gisquet, and P. Onno, "CE3-5.1: On crosscomponent linear model simplification," 12th JVET Meeting, Doc. JVET-L0191, Oct. 2018.

[10] VVC Test Model (VTM), https://vcgit.hhi.fraunhofer.de/jvet/ VVCSoftware_VTM/tags

[11] F. Bossen, J. Boyce, X. Li, V. Seregin, and K. Suhring, "JVET common test conditions and software reference configurations for SDR video," 12th JVET Meeting, Doc. JVET-L1010, Oct. 2018. 